\title{
Short and efficient method for the preparation of furo[3,2-f] quinoline
}

\author{
Mercedesz Törincsi, Pál Kolonits, Endre Pálosi, Melinda Fekete, and Lajos Novák* \\ Department of Organic Chemistry and Technology, Budapest University of Technology and \\ Economics, Research Group for Alkaloid Chemistry, Hungarian Academy of Sciences, \\ Gellért tér 4, 1111 Budapest, Hungary \\ E-mail:Inovak@mail.bme.hu
}

Dedicated to Professor Csaba Szántay on his $80^{\text {th }}$ birthday

\begin{abstract}
Furo[3,2-f]quinolines 5 were prepared by reacting the sodium salt of quinolin-6-ol and allyl bromides $\mathbf{2}$. The allyl aryl ethers 3 formed were then thermally rearranged under standard or microwave conditions. Acid-catalyzed cyclization of the products 4 afforded the title compounds.
\end{abstract}

Keywords: Furo[3,2-f]quinoline, allyl aryl ethers, Claisen rearrangement, ring closure, sonochemical conditions, pyrano[3,2-f]quinoline

\section{Introduction}

Furo[3,2-f]quinolines have not received much attention. Only three papers have so far been published dealing with the the preparation of this ring system. B. B. Dey and T. R. Seshadri prepared the furoquinoline skeleton by the thermal decomposition of a quinolinopyrone derivative. ${ }^{1} \mathrm{R}$. Royer et $a l .{ }^{2}$ described the synthesis of substituted furo[3,2-f]quinolines by the rearrangement of allylaryl ether followed by cyclization. Later on, M. Natsume et al. ${ }^{3}$ isolated the furoquinoline as by-product of their duocarmycin SA's synthesis.

Recently we have developed an effective method for the preparation of furo[2,3$f$ jisoquinolines by aromatic Claisen rearrangement and subsequent cyclization. ${ }^{4}$ Following these synthetic efforts toward the preparation of novel heterocyclic compounds which might be useful intermediates for the development of molecules of pharmaceutical or biological interest, we planned the elaboration of new synthesis generally applicable for the preparation of furo[3,2$f$ ]quinolines. We present here a method for the preparation of furoquinolines 5a-c and their cycloalkano analogues $\mathbf{5 d , e . ~}$ 


\section{Results and Discussion}

Ethers of quinolin-6-ol 3a-d were synthesized by reaction of the sodium salt of quinolinol (1) with the appropriate alkyl bromide 2a-d in good to acceptable yields (Scheme 1, Table 1, entries 1-4).

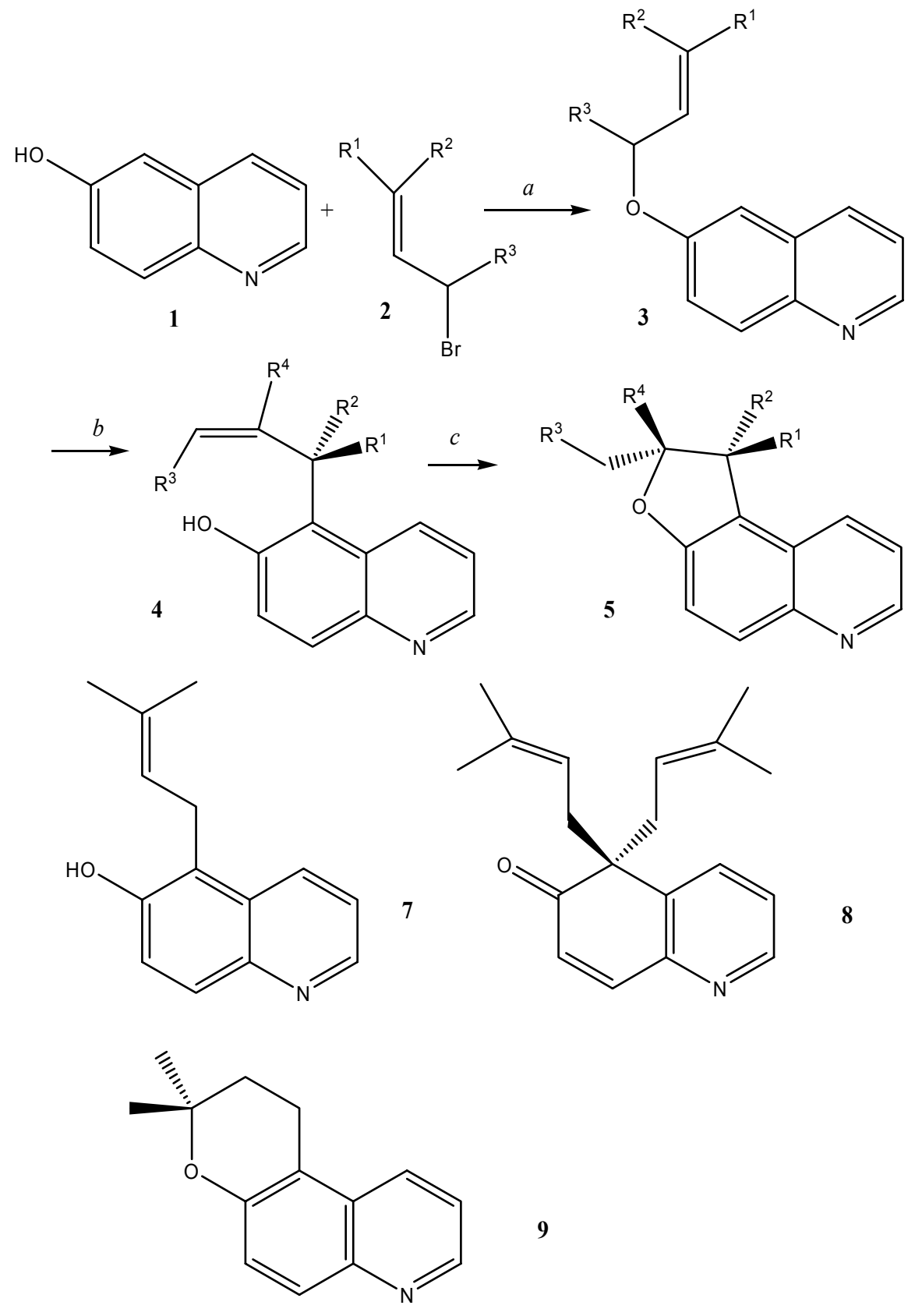

Scheme 1. Reagent and conditions: (a) $\mathrm{NaH}$, DME, r.t.; (b) microwave oven, $175^{\circ} \mathrm{C}$; or chlorobenzene, reflux; (c) $\mathrm{H}_{2} \mathrm{SO}_{4}, 100^{\circ} \mathrm{C}$. 
The allyl ether 3a was subjected to thermal [3,3] rearrangement in a microwave oven to afford 4a. ${ }^{5}$ Acid-catalyzed intramolecular cyclization of the latter afforded the known furo[3,2] quinoline $\mathbf{5} \mathbf{a}^{2}$ (entries 5 and 10).

Starting with compound $\mathbf{3 b}$, the microwave assisted rearrangement gave two products $\mathbf{4 b}$ and $\mathbf{4 c}$ (entry 6). Compound $\mathbf{4 b}$ was formed in the normal Claisen rearrangement. ${ }^{6}$ The unexpected product took its origin from consecutive rearrangement reactions (Scheme 2). Namely, [3,3]sigmatropic rearrangement of ether $\mathbf{3 b}$ yielded intermediate $\mathbf{4 c}$ which then underwent a homo[1,5]-H shift to afford compound 6. Further [1,5]-H migration on this intermediate led to the formation of $\mathbf{4 c} .^{7}$

Table 1. Compounds prepared by the rearrangement of 3a-d and subsequent cyclization

\begin{tabular}{cccccccccc}
\hline Entry & Substrate & Product & $\mathrm{R}^{1}$ & $\mathrm{R}^{2}$ & $\mathrm{R}^{3}$ & $\mathrm{R}^{4}$ & $\begin{array}{r}\text { Temp } \\
\left({ }^{\circ} \mathrm{C}\right)\end{array}$ & $\begin{array}{c}\text { Time } \\
(\mathrm{h})\end{array}$ & $\begin{array}{c}\text { Yield }^{\mathrm{a}} \\
(\%)\end{array}$ \\
\hline 1 & $\mathbf{2 a}$ & $\mathbf{3 a}$ & $\mathrm{H}$ & $\mathrm{H}$ & $\mathrm{H}$ & - & r.t. & 24 & 69 \\
2 & $\mathbf{2 b}$ & $\mathbf{3 b}$ & $\mathrm{Me}$ & $\mathrm{Me}$ & $\mathrm{H}$ & - & r.t. & 24 & 83 \\
3 & $\mathbf{2 c}$ & $\mathbf{3 c}$ & $\mathrm{H}$ & $\left(\mathrm{CH}_{2}\right)_{3}$ & - & r.t. & 24 & 46 \\
4 & $\mathbf{2 d}$ & $\mathbf{3 d}$ & $\mathrm{H}$ & $\left(\mathrm{CH}_{2}\right)_{4}$ & - & r.t. & 24 & 38 \\
5 & $\mathbf{3 a}$ & $\mathbf{4 a}$ & $\mathrm{H}$ & $\mathrm{H}$ & $\mathrm{H}$ & $\mathrm{H}$ & $175^{\mathrm{b}}$ & 8 & 49.5 \\
6 & $\mathbf{3 b}$ & $\mathbf{4 b}$ & $\mathrm{Me}$ & $\mathrm{Me}$ & $\mathrm{H}$ & $\mathrm{H}$ & $175^{\mathrm{b}}$ & 10 & 44 \\
& & $\mathbf{4 c}$ & $\mathrm{Me}$ & $\mathrm{H}$ & $\mathrm{H}$ & $\mathrm{Me}$ & & & 12 \\
7 & $\mathbf{2 b}$ & $\mathbf{3 b}$ & $\mathrm{Me}$ & $\mathrm{Me}$ & $\mathrm{H}$ & $\mathrm{H}$ & r.t. & 16 & 16.8 \\
& & $\mathbf{7}$ & $\mathrm{H}$ & $\mathrm{H}$ & $\mathrm{Me}$ & $\mathrm{Me}$ & & & 26.2 \\
& & $\mathbf{8}$ & - & - & - & - & & & 32.4 \\
8 & $\mathbf{3 c}$ & $\mathbf{4 e}$ & $\mathrm{H}$ & $\left(\mathrm{CH}_{2}\right)_{3}$ & $\mathrm{H}$ & $132^{\mathrm{c}}$ & 100 & 70 \\
9 & $\mathbf{3 d}$ & $\mathbf{4 f}$ & $\mathrm{H}$ & $\left(\mathrm{CH}_{2}\right)_{4}$ & $\mathrm{H}$ & $132^{\mathrm{c}}$ & 100 & 38 \\
10 & $\mathbf{4 a}$ & $\mathbf{5 a}$ & $\mathrm{H}$ & $\mathrm{H}$ & $\mathrm{H}$ & $\mathrm{Me}$ & 100 & 1.5 & 35 \\
11 & $\mathbf{4 b}$ & $\mathbf{5 b}$ & $\mathrm{Me}$ & $\mathrm{Me}$ & $\mathrm{H}$ & $\mathrm{H}$ & 100 & 1 & 42.2 \\
12 & $\mathbf{4 c}$ & $\mathbf{5 c}$ & $\mathrm{Me}$ & $\mathrm{H}$ & $\mathrm{Me}$ & $\mathrm{H}$ & 100 & 1 & 40 \\
13 & $\mathbf{7}$ & $\mathbf{9}$ & - & - & - & - & 100 & 1 & 54 \\
14 & $\mathbf{4 d}$ & $\mathbf{5 d}$ & $\mathrm{H}$ & $\left(\mathrm{CH}_{2}\right)_{3}$ & $\mathrm{H}$ & 100 & 1.5 & 75 \\
15 & $\mathbf{4 e}$ & $\mathbf{5 e}$ & $\mathrm{H}$ & $\left(\mathrm{CH}_{2}\right)_{4}$ & $\mathrm{H}$ & 100 & 1.5 & 74 \\
\hline
\end{tabular}

${ }^{\mathrm{a}}$ Isolated and unoptimized yields; ${ }^{\mathrm{b}}$ in microwave oven; ${ }^{\mathrm{c}}$ in chlorobenzene.

Having the above result, we tried to synthesize compounds $\mathbf{3 b}$ and $\mathbf{4 b}$ with a phase transfer catalyzed reaction. Interestingly, the quaternary ammonium salt catalyzed reaction of quinolin-6ol 1 with prenyl bromide (2b) afforded three compounds (entry 7). Besides the expected ether $\mathbf{3 b}$, compounds $\mathbf{7}$ and $\mathbf{8}$ were isolated as major products. The formation of these new compounds is probably the result of direct aromatic electrophilic substitutions. 


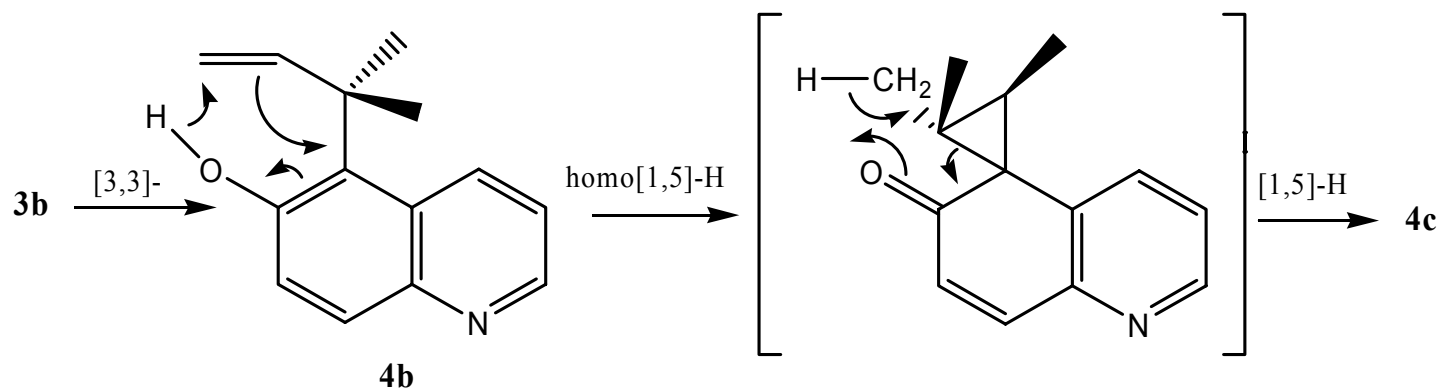

\section{Scheme 2}

Acid-catalyzed intramolecular cyclization of $\mathbf{4 b}$ gave furoquinoline $\mathbf{5 b}$ in moderate yield. Likewise, compound $\mathbf{4 c}$ yielded $\mathbf{5} \mathbf{c}$ by acid $\left(\mathrm{H}_{2} \mathrm{SO}_{4}\right.$ or $\left.\mathrm{HClO}_{4}\right)$ promoted ring closure (entries 11 and 12).

Treatment of the dimethylallyl derivative 7 with sulfuric acid provided pyrano [3,2-f] quinoline derivative 9 (entry 13).

Due to their sensitivity at the temperature of the microwave oven, compounds $\mathbf{3 c}$ and $\mathbf{3 d}$ were submitted to thermal rearrangement in boiling chlorobenzene. This protocol was especially effective for the preparation of $\mathbf{4 d}$, but a moderate result was obtained for the synthesis of $\mathbf{4 e}$ (entries 8 and 9). Acid-catalyzed cyclization of $\mathbf{4 d}$ and $\mathbf{4 e}$ yielded $\mathbf{5 d}$ and $\mathbf{5 e}$ (respectively), as an approximately 4:1 mixture of trans and cis stereoisomers (entries 14 and 15). Small amounts of these mixtures were separated by preparative HPLC and the stereochemistry of isomers was established by ${ }^{1} \mathrm{H}$ and ${ }^{13} \mathrm{C}$ NMR studies. For example, in the cis-fused isomer of $\mathbf{5 d}$ we saw a NOE interaction between the $7 \mathrm{a}$ proton and $11 \mathrm{a}$ proton (4.82 and 3.45, respectively). This interaction was absent in the spectrum of the corresponding trans-isomer. For $\mathbf{5 e}$, on the bases of $\gamma$-effect in chemical shift between $\mathrm{C}_{8}$ and $\mathrm{C}_{12}$ in the spectrum the cis-isomer was identified. This correlation is in concord with our earlier findings in the series of furo[2,3-f]isoquinolines. ${ }^{4}$

In summary, we have developed a general and efficient method for the preparation of [3,2$f$ qquinoline derivatives 5a-e from quinolin-6-ol (1) and allyl bromides 2a-d. This process involves the thermal rearrangement of ethers 3a-d, followed by acid-catalyzed intramolecular cyclization of the products 4a-e. This synthesis using the readily available starting compounds seems to provide a powerful methodology for the construction of furo-condensed quinoline derivatives.

\section{Experimental Section}

General Procedures. Solvents were used as received from commercial vendors and no further attempts were made to purify or dry them. Mps were determined on a Büchi apparatus and are uncorrected. ${ }^{1} \mathrm{H}$ and ${ }^{13} \mathrm{C}$ NMR spectra were obtained on a Bruker DRX-500 spectrometer. All NMR spectra are reported in ppm relative to TMS. IR spectra were measured on a Specord 2000 
spectrometer. Merck precoated silica gel $60 \mathrm{~F}_{254}$ plates were used for TLC and Kieselgel 60 for column chromatography. Solvent were mixed on a v/v basis. HPLC chromatographic analyses and separation were performed with a Waters 600 equipped with a photodiode array detector 990. Stationary phase for compound 5e was Supelcosil ${ }^{\mathrm{TM}}$ SPLC-18-DB, 250x10 mm, eluens $\mathrm{MeOH} / \mathrm{H}_{2} \mathrm{O} / \mathrm{H}_{3} \mathrm{PO}_{4}$ 4:6:0.05. For compound 5d stationary phase was Waters Symmetry $\mathrm{C}_{18}$, $150 \times 3.9 \mathrm{~mm}$. For the separation of isomers of $\mathbf{5 f}$ we worked on Supelcosil ${ }^{\mathrm{TM}}$ PLC-18 column, 250x21.2 mm, eluens $\mathrm{MeOH} / 3 \%$ tartaric acid solution $/ \mathrm{H}_{3} \mathrm{PO}_{4}$ 4:6:0.02.

Microwave accelerated reactions were conducted in an CEM Focused Microwave ${ }^{\mathrm{TM}}$ Synthesis System (CEM Corporation, Metthews, NC,USA).

3-Bromocyclohex-1-ene $(\mathbf{2 c})^{8}$ and 3-bromocyclohept-1-ene $(\mathbf{2 d})^{9}$ were prepared by literature procedures.

\section{Preparation of ethers 3. General procedure}

To a cold stirred suspension of $\mathrm{NaH}$ (24 mmol, 63.7\% in mineral oil) in DME (10 mL) a solution of quinolin-6-ol (1: $2.23 \mathrm{~g}, 15.4 \mathrm{mmol})$ in DME (200 mL) was added dropwise and the resultant mixture was stirred at $0{ }^{\circ} \mathrm{C}$ for $1.5 \mathrm{~h}$. To this mixture the appropriate bromide (2: $\left.23 \mathrm{mmol}\right)$ was then added and stirring was continued at r.t. for $24 \mathrm{~h}$. The reaction mixture was quenched with sat. aq. $\mathrm{NaCl}(400 \mathrm{~mL})$ and extracted with EtOAc $(3 \times 100 \mathrm{~mL})$. The combined organic layers were washed with $1 \mathrm{~N} \mathrm{NaOH}$ solution and $\mathrm{H}_{2} \mathrm{O}$ and then dried over $\mathrm{MgSO}_{4}$. The solvent was removed under reduced pressure and the residue was purified by column chromatography on silica gel $\left(\mathrm{CH}_{2} \mathrm{Cl}_{2}\right.$ - acetone $\left.4: 1\right)$.

6-Allyloxyquinoline (3a). Yield: $69 \%$; brown oil; $R_{f}=0.77\left(\mathrm{CH}_{2} \mathrm{Cl}_{2}-\right.$ acetone $\left.2: 1\right)$. ${ }^{1} \mathrm{H}$ NMR $\left(\mathrm{CDCl}_{3}\right): \delta=4.66\left(\mathrm{~d}, J=5.4 \mathrm{~Hz}, 2 \mathrm{H}, \mathrm{CH}_{2}\right), 5.34(\mathrm{dd}, J=10.6$ and $1.1 \mathrm{~Hz}, 1 \mathrm{H},=\mathrm{CH}), 5.47(\mathrm{dd}, J=$ 17.2 and $1.2 \mathrm{~Hz}, 1 \mathrm{H},=\mathrm{CH}), 6.12(\mathrm{~m}, 1 \mathrm{H},=\mathrm{CH}), 7.08\left(\mathrm{~d}, J=2.7 \mathrm{~Hz}, 1 \mathrm{H}, \mathrm{C}_{5}-\mathrm{H}\right), 7.34(\mathrm{dd}, J=8.2$ and $\left.1.2 \mathrm{~Hz}, 1 \mathrm{H}, \mathrm{C}_{3}-\mathrm{H}\right), 7.40\left(\mathrm{dd}, J=9.1\right.$ and $\left.2.7 \mathrm{~Hz}, 1 \mathrm{H}, \mathrm{C}_{7}-\mathrm{H}\right), 7.99\left(\mathrm{~d}, J=9.1 \mathrm{~Hz}, 1 \mathrm{H}, \mathrm{C}_{8}-\mathrm{H}\right)$, $8.02\left(\mathrm{~m}, 1 \mathrm{H}, \mathrm{C}_{4}-\mathrm{H}\right), 8.77\left(\mathrm{dd}, J=4.2\right.$ and $\left.1.6 \mathrm{~Hz}, 1 \mathrm{H}, \mathrm{C}_{2}-\mathrm{H}\right)$.

${ }^{13} \mathrm{C}$ NMR $\left(\mathrm{CDCl}_{3}\right): \delta=69.07$ (C-2'), 106.38 (C-5), 118.04 (C-4'), 121.37 (C-3), 122.51 (C-6), 129.25 (C-4a), 130.94 (C-7), 132.84 (C-3'), 134.81 (C-4), 144.48 (C-7a), 148.06 (C-2), 156.67 (C-6).

6-(3-Methylbut-2-en-1-yloxy)quinoline (3b). Yield: 83\%; brown oil; $R_{f}=0.56\left(\mathrm{CHCl}_{3}-\right.$ acetone 10:1). ${ }^{1} \mathrm{H} \mathrm{NMR}\left(\mathrm{CDCl}_{3}\right): \delta=1.78\left(\mathrm{~s}, 3 \mathrm{H}, \mathrm{CH}_{3}\right), 1.82\left(\mathrm{~s}, 3 \mathrm{H}, \mathrm{CH}_{3}\right), 4.62(\mathrm{~d}, J=6.6 \mathrm{~Hz}$, $\left.2 \mathrm{H}, \mathrm{CH}_{2}\right), 5.56\left(\mathrm{t}, J=6.6 \mathrm{~Hz}, 1 \mathrm{H}, \mathrm{C}_{2},-\mathrm{H}\right), 7.08\left(\mathrm{~d}, J=2.5 \mathrm{~Hz}, 1 \mathrm{H}, \mathrm{C}_{5}-\mathrm{H}\right), 7.34(\mathrm{dd}, J=8.2$ and 4.2 $\left.\mathrm{Hz}, 1 \mathrm{H}, \mathrm{C}_{3}-\mathrm{H}\right), 7.39\left(\mathrm{dd}, J=9.2\right.$ and $\left.2.5 \mathrm{~Hz}, 1 \mathrm{H}, \mathrm{C}_{7}-\mathrm{H}\right), 8.01\left(\mathrm{~d}, J=9.6 \mathrm{~Hz}, 1 \mathrm{H}, \mathrm{C}_{8}-\mathrm{H}\right), 8.03$ (d, $\left.J=9.8 \mathrm{~Hz}, 1 \mathrm{H}, \mathrm{C}_{4}-\mathrm{H}\right) .{ }^{13} \mathrm{C} \mathrm{NMR}\left(\mathrm{CDCl}_{3}\right): \delta=18.21\left(\mathrm{CH}_{3}\right), 25.69\left(\mathrm{CH}_{3}\right), 64.90\left(\mathrm{C}-1{ }^{\prime}\right), 105.74(\mathrm{C}-$ 5), 118.86 (C-2'), 120.93 (C-3), 122.41 (C-7), 128.93 (C-4a), 130.28 (C-8), 134.52 (C-4), 138.29 (C-3'), 143.75 (C-8a), 147.25 (C-2), 156.54 (C-6). Anal. Calcd for $\mathrm{C}_{14} \mathrm{H}_{15} \mathrm{NO}$ : C, 78.84; H, 7.09; $\mathrm{N}, 6.57$. Found: C, 78.57; H, 7.12; N, 6.32.

6-(Cyclohex-2-en-1-yloxy)quinoline (3c). Yield: 46\%; yellow solid mp $65-69{ }^{\circ} \mathrm{C} ; R_{f}=0.28$ (hexane - EtOAc 4:1). ${ }^{1} \mathrm{H}$ NMR $\left(\mathrm{CDCl}_{3}\right): \delta=1.78\left(\mathrm{~m}, 1 \mathrm{H}, \mathrm{C}_{5}, \mathrm{H}\right), 1.87\left(\mathrm{~m}, 1 \mathrm{H}, \mathrm{C}_{5},-\mathrm{H}\right), 1.95(\mathrm{~m}$, $\left.1 \mathrm{H}, \mathrm{C}_{6},-\mathrm{H}\right), 2.03\left(\mathrm{~m}, 1 \mathrm{H}, \mathrm{C}_{6},-\mathrm{H}\right), 2.06\left(\mathrm{~m}, 1 \mathrm{H}, \mathrm{C}_{4},-\mathrm{H}\right), 2.17\left(\mathrm{~m}, 1 \mathrm{H}, \mathrm{C}_{4},-\mathrm{H}\right), 4.95\left(\mathrm{~m}, 1 \mathrm{H}, \mathrm{C}_{1},-\mathrm{H}\right)$, 
$5.94\left(\mathrm{~m}, 1 \mathrm{H}, \mathrm{C}_{2},-\mathrm{H}\right), 6.02\left(\mathrm{~m}, 1 \mathrm{H}, \mathrm{C}_{3},-\mathrm{H}\right), 7.12\left(\mathrm{~d}, J=2.6 \mathrm{~Hz}, 1 \mathrm{H}, \mathrm{C}_{5}-\mathrm{H}\right), 7.33(\mathrm{dd}, J=8.3$ and 4.3 $\left.\mathrm{Hz}, 1 \mathrm{H}, \mathrm{C}_{3}-\mathrm{H}\right), 7.38\left(\mathrm{dd}, J=9.2\right.$ and $\left.2.6 \mathrm{~Hz}, 1 \mathrm{H}, \mathrm{C}_{7}-\mathrm{H}\right), 8.01\left(\mathrm{~d}, J=9.2 \mathrm{~Hz}, 1 \mathrm{H}, \mathrm{C}_{8}-\mathrm{H}\right), 8.03$ (d, $\left.J=9.2 \mathrm{~Hz}, 1 \mathrm{H}, \mathrm{C}_{4}-\mathrm{H}\right), 8.75\left(\mathrm{dd}, J=4.3\right.$ and $\left.1 \mathrm{~Hz}, 1 \mathrm{H}, \mathrm{C}_{2}-\mathrm{H}\right) .{ }^{13} \mathrm{C} \mathrm{NMR}\left(\mathrm{CDCl}_{3}\right): \delta=19.11\left(\mathrm{C}-5^{\prime}\right)$, 25.19 (C-4'), 28.25 (C-6'), 71.13 (C-1'), 107.26 (C-5), 121.08 (C-3), 123.14 (C-7), 125.55 (C2'), 129.11 (C-4a), 130.59 (C-8), 132.39 (C-3'), 134.60 (C-4), 143.83 (C-8a), 147.45 (C-2'), 155.61 (C-6). Anal. Calcd for $\mathrm{C}_{15} \mathrm{H}_{15} \mathrm{NO}$ : C, 79.97; H, 6.71; N, 6.22. Found: C, 79.71; H, 6.53; N, 6.34 .

6-(Cyclohept-2-en-1-yloxy)quinoline (3d). Yield: 38\%; brown oil ; $R_{f}=0.71\left(\mathrm{CH}_{2} \mathrm{Cl}_{2}\right.$ - acetone 2:1). ${ }^{1} \mathrm{H}$ NMR $\left(\mathrm{CDCl}_{3}\right): \delta=1.45\left(\mathrm{~m}, 1 \mathrm{H}, \mathrm{C}_{5},-\mathrm{H}\right), 1.72\left(\mathrm{~m}, 1 \mathrm{H}, \mathrm{C}_{6},-\mathrm{H}\right), 1.76\left(\mathrm{~m}, 1 \mathrm{H}, \mathrm{C}_{5},-\mathrm{H}\right), 1.83$ (m, 1H, C $\left.\mathrm{C}_{7}, \mathrm{H}\right), 2.07\left(\mathrm{~m}, 1 \mathrm{H}, \mathrm{C}_{6},-\mathrm{H}\right), 2.10\left(\mathrm{~m}, 1 \mathrm{H}, \mathrm{C}_{7},-\mathrm{H}\right), 2.20\left(\mathrm{~m}, 1 \mathrm{H}, \mathrm{C}_{4},-\mathrm{H}\right), 2.27$ (m, 1H, C ${ }_{4},-$ $\mathrm{H}), 5.02\left(\mathrm{~m}, 1 \mathrm{H}, \mathrm{C}_{1},-\mathrm{H}\right), 5.84\left(\mathrm{~m}, 1 \mathrm{H}, \mathrm{C}_{2},-\mathrm{H}\right), 5.92\left(\mathrm{~m}, 1 \mathrm{H}, \mathrm{C}_{3},-\mathrm{H}\right), 7.01\left(\mathrm{~d}, J=2.5 \mathrm{~Hz}, 1 \mathrm{H}, \mathrm{C}_{5^{-}}\right.$ $\mathrm{H}), 7.32\left(\mathrm{dd}, J=8.3\right.$ and $\left.4.2 \mathrm{~Hz}, 1 \mathrm{H}, \mathrm{C}_{3}-\mathrm{H}\right), 7.37\left(\mathrm{dd}, J=9.2\right.$ and $\left.2.5 \mathrm{~Hz}, 1 \mathrm{H}, \mathrm{C}_{7}-\mathrm{H}\right), 8.00(\mathrm{~d}, J=$ $\left.9.0 \mathrm{~Hz}, 1 \mathrm{H}, \mathrm{C}_{8}-\mathrm{H}\right), 8.02\left(\mathrm{~d}, J=7.0 \mathrm{~Hz}, 1 \mathrm{H}, \mathrm{C}_{4}-\mathrm{H}\right), 8.75\left(\mathrm{~d}, J=3.8 \mathrm{~Hz}, 1 \mathrm{H}, \mathrm{C}_{2}-\mathrm{H}\right) .{ }^{13} \mathrm{C}$ NMR $\left(\mathrm{CDCl}_{3}\right): \delta=26.48$ (C-5'), 27.49 (C-6'), 28.58 (C-4'), 77.65 (C-1'), 107.44 (C-5), 121.25 (C-3), 123.18 (C-7), 129.30 (C-4a), 130.85 (C-8), 131.44 (C-3’), 134.84 (C-4), 135.16 (C-2'), 144.17

(C-8a), 147.76 (C-2), 155.73 (C-6). Anal. Calcd for $\mathrm{C}_{16} \mathrm{H}_{17} \mathrm{NO}: \mathrm{C}, 80.30 ; \mathrm{H}, 7.16$; $\mathrm{N}$, 5.85.Found: C, 80.02; H, 7.12; N, 6.01.

\section{5-Allylquinolin-6-ol (4a)}

3a $(1.2 \mathrm{~g}, 6.4 \mathrm{mmol})$ was heated at $175^{\circ} \mathrm{C}$ for $8 \mathrm{~h}$ in a microwave oven. After cooling, the light brown solid was treated with $\mathrm{CH}_{2} \mathrm{Cl}_{2}$ and the precipitated crystalls were collected by filtration. Yield: $0.59 \mathrm{~g} \mathrm{(49.5 \% );} \mathrm{mp} \mathrm{158-162}{ }^{\circ} \mathrm{C}$ [lit., $\left.{ }^{2} 155^{\circ} \mathrm{C}\right] ; R_{f}=0.44\left(\mathrm{CH}_{2} \mathrm{Cl}_{2}\right.$ - acetone 2:1).

${ }^{1} \mathrm{H}$ NMR $\left(\mathrm{CDCl}_{3}\right): \delta=3.73\left(\mathrm{~d}, J=5.7 \mathrm{~Hz}, 2 \mathrm{H}, \mathrm{CH}_{2}\right), 4.93(\mathrm{~m}, 2 \mathrm{H},=\mathrm{CH}), 5.95(\mathrm{~m}, 1 \mathrm{H}, \mathrm{HC}=)$, $7.41\left(\mathrm{dd}, J=9\right.$ and $\left.4 \mathrm{~Hz}, 1 \mathrm{H}, \mathrm{C}_{3}-\mathrm{H}\right), 7.42\left(\mathrm{~d}, J=9 \mathrm{~Hz}, 1 \mathrm{H}, \mathrm{C}_{7}-\mathrm{H}\right), 7.78\left(\mathrm{~d}, J=9 \mathrm{~Hz}, 1 \mathrm{H}, \mathrm{C}_{8}-\mathrm{H}\right)$, $8.26\left(\mathrm{~d}, J=8.5 \mathrm{~Hz}, 1 \mathrm{H}, \mathrm{C}_{4}-\mathrm{H}\right), 8.66\left(\mathrm{~d}, J=3 \mathrm{~Hz}, 1 \mathrm{H}, \mathrm{C}_{2}-\mathrm{H}\right), 9.88(\mathrm{~s}, 1 \mathrm{H}, \mathrm{OH}) .{ }^{13} \mathrm{C} \mathrm{NMR}\left(\mathrm{CDCl}_{3}\right)$ : $\delta=28.46\left(\mathrm{CH}_{2}\right), 115.16(=\mathrm{C}), 116.80(\mathrm{C}-5), 121.20(\mathrm{C}-3), 121.49(\mathrm{C}-7), 128.23(\mathrm{C}-4 \mathrm{a}), 128.75$ (C-8), 131.33 (C-4), 136.79 (C=), 143.56 (C-C-8a), 146.72 (C-2), 152.59 (C-6).

\section{5-(2-Methylbut-3-en-2-yl)quinolin-6-ol (4b) and 5-(3-methylbut-3-en-2-yl)quinolin-6-ol (4c)}

3b $(1.0 \mathrm{~g}, 4.7 \mathrm{mmol})$ was heated at $175{ }^{\circ} \mathrm{C}$ for $10 \mathrm{~h}$ in a microwave oven. After cooling, the reaction mixture was dissolved in a mixture of $\mathrm{CH}_{2} \mathrm{Cl}_{2}$ - acetone (5:2) and purified by column chromatography to yield $0.44 \mathrm{~g}$ of $\mathbf{4 b}(44 \%)$ and $0.12 \mathrm{~g}$ of $\mathbf{4 c}(12 \%)$.

Compound 4b. Brown crystalline solid; mp 132-136 ${ }^{\circ} \mathrm{C} ; R_{f}=0.18\left(\mathrm{CHCl}_{3}-\right.$ acetone $\left.10: 1\right) .{ }^{1} \mathrm{H}$ $\operatorname{NMR}\left(\mathrm{CDCl}_{3}\right): \delta=1.80\left(\mathrm{~s}, 6 \mathrm{H}, 2 \mathrm{CH}_{3}\right), 5.20(\mathrm{~m}, 2 \mathrm{H},=\mathrm{CH}), 6.44(\mathrm{~m}, 1 \mathrm{H}, \mathrm{HC}=), 7.28\left(\mathrm{~m}, 2 \mathrm{H}, \mathrm{C}_{3}-\right.$ $\mathrm{H}$ and $\left.\mathrm{C}_{7}-\mathrm{H}\right), 7.84\left(\mathrm{~d}, J=9 \mathrm{~Hz}, 1 \mathrm{H}, \mathrm{C}_{8}-\mathrm{H}\right), 8.67\left(\mathrm{~d}, J=3.7 \mathrm{~Hz}, 1 \mathrm{H}, \mathrm{C}_{2}-\mathrm{H}\right), 8.79(\mathrm{~d}, J=8.9 \mathrm{~Hz}, 1 \mathrm{H}$, $\left.\mathrm{C}_{4}-\mathrm{H}\right) .{ }^{13} \mathrm{C}$ NMR $\left(\mathrm{CDCl}_{3}\right): \delta=29.84\left(2 \mathrm{CH}_{3}\right), 42.65$ (C-2'), 110.95 (C-4'), $119.13(\mathrm{C}-3), 123.23$ (C-5), 124.33 (C-7), 128.89 (C-4a), 129.28 (C-8), 135.36 (C-4), 144.61 (C-8a), 145.59 (C-2), 151.22 (C-3'), 153.33 (C-6). Anal. Calcd for $\mathrm{C}_{14} \mathrm{H}_{15} \mathrm{NO}$ : C, 78.84; H, 7.09; N, 6.57.Found: C, 78.60; H, 7.17; N, 6.35.

Compound 4c. Light brown crystals; mp 172-176 ${ }^{\circ} \mathrm{C} ; R_{f}=0.26\left(\mathrm{CHCl}_{3}-\right.$ acetone $\left.10: 1\right) .{ }^{1} \mathrm{H}$ $\operatorname{NMR}\left(\mathrm{CDCl}_{3}\right): \delta=1.55\left(\mathrm{~d}, J=6.2 \mathrm{~Hz}, 3 \mathrm{H}, \mathrm{CH}_{3}\right), 1.71\left(\mathrm{~s}, 3 \mathrm{H}, \mathrm{CH}_{3}\right), 4.32\left(\mathrm{q}, J=6.5 \mathrm{~Hz}, 1 \mathrm{H}, \mathrm{C}_{2}\right.$ 'H), $5.22\left(\mathrm{~d}, J=0.9 \mathrm{~Hz}, 1 \mathrm{H}, \mathrm{C}_{4},-\mathrm{H}\right), 5.29\left(\mathrm{~s}, 1 \mathrm{H}, \mathrm{C}_{4},-\mathrm{H}\right), 7.34\left(\mathrm{~d}, J=9.1 \mathrm{~Hz}, 1 \mathrm{H}, \mathrm{C}_{7}-\mathrm{H}\right), 7.37$ (dd, 
$J=8.7$ and $\left.4.2 \mathrm{~Hz}, 1 \mathrm{H}, \mathrm{C}_{3}-\mathrm{H}\right), 7.89\left(\mathrm{~d}, J=9.1 \mathrm{~Hz}, 1 \mathrm{H}, \mathrm{C}_{8}-\mathrm{H}\right), 8.47\left(\mathrm{~d}, J=8.6 \mathrm{~Hz}, 1 \mathrm{H}, \mathrm{C}_{4}-\mathrm{H}\right), 8.74$ $\left(\mathrm{dd}, J=4.2\right.$ and $\left.1.2 \mathrm{~Hz}, 1 \mathrm{H}, \mathrm{C}_{2}-\mathrm{H}\right) .{ }^{13} \mathrm{C} \mathrm{NMR}\left(\mathrm{CDCl}_{3}\right): \delta=17.34\left(\mathrm{C}-1\right.$ ') $, 22.77\left(\mathrm{CH}_{3}\right), 37.48(\mathrm{C}-$ 2'), 111.43 (C-4'), 120.67 (C-5), 120.81 (C-3), 122.86 (C-7), 128.37 (C-4a), 129.08 (C-8), 131.35 (C-4), 144.09 (C-8a), 146.53 (C-2), 150.06 (C-3'), 153.36 (C-6). Anal. Calcd for $\mathrm{C}_{14} \mathrm{H}_{15} \mathrm{NO}$ : C, 78.84; H, 7.09; N, 6.57.Found: C, 78.66; H, 6.92; N, 6.38.

\section{5-(3-Methylbut-2-en-1-yl)quinolin-6-ol (7) and 5,5-bis(3-methylbut-2-en-1-yl)quinolin- 6(5H)-one (8)}

To a stirred mixture of 1 (1.0 g, $6.9 \mathrm{mmol}), \mathrm{KOH}(24.4 \mathrm{~mL} 12.5 \%$ solution), and triethylbenzylammonium chloride $(0.73 \mathrm{~g}, 4 \mathrm{mmol})$ in toluene $(70 \mathrm{~mL})$ was added $\mathbf{2 b}(1.87 \mathrm{~g}$, $12.6 \mathrm{mmol}$ ), and stirring was continued at r.t. for $16 \mathrm{~h}$. The reaction mixture was extracted with EtOAc $(3 \times 50 \mathrm{~mL})$, the combined extracts were washed with sat. aq $\mathrm{NaCl}$, and dried over $\mathrm{MgSO}_{4}$. Evaporation of the solvent under reduced pressure provided a mixture of three compounds which was separated by column chromatography on silica gel $\left(\mathrm{CHCl}_{3}-\right.$ acetone $10: 1)$ to give $3 \mathbf{b}(0.25 \mathrm{~g}, 16.8 \%), 7(0.38 \mathrm{~g}, 26.2 \%)$, and $\mathbf{8}(0.47 \mathrm{~g}, 32.4 \%)$.

Compound 7. Brown crystals; mp $154-158{ }^{\circ} \mathrm{C}\left(\mathrm{EtOH}-\mathrm{H}_{2} \mathrm{O} 3: 2\right) ; R_{f}=0.21\left(\mathrm{CHCl}_{3}\right.$ - acetone 10:1). IR (KBr): $3450(\mathrm{~s}, \mathrm{OH}) \mathrm{cm}^{-1} .{ }^{1} \mathrm{H} \mathrm{NMR}\left(\mathrm{CDCl}_{3}\right): \delta=1.69\left(\mathrm{~s}, 3 \mathrm{H}, \mathrm{CH}_{3}\right), 1.88\left(\mathrm{~s}, 3 \mathrm{H}, \mathrm{CH}_{3}\right)$, $3.76\left(\mathrm{~d}, J=6 \mathrm{~Hz}, 2 \mathrm{H}, \mathrm{C}_{1}\right.$,-H), 3.83 (br s, $\left.1 \mathrm{H}, \mathrm{OH}\right), 5.19$ (t, $\left.J=6.5 \mathrm{~Hz}, 1 \mathrm{H}, \mathrm{C}_{2},-\mathrm{H}\right), 7.36$ (t, $J=6.5$ $\left.\mathrm{Hz}, 1 \mathrm{H}, \mathrm{C}_{3}-\mathrm{H}\right), 7.38\left(\mathrm{~d}, J=9 \mathrm{~Hz}, 1 \mathrm{H}, \mathrm{C}_{7}-\mathrm{H}\right), 7.80$ (d, $\left.J=9 \mathrm{~Hz}, 1 \mathrm{H}, \mathrm{C}_{8}-\mathrm{H}\right), 8.27$ (d, $J=9 \mathrm{~Hz}, 1 \mathrm{H}$, $\left.\mathrm{C}_{4}-\mathrm{H}\right), 8.63\left(\mathrm{~d}, J=5 \mathrm{~Hz}, 1 \mathrm{H}, \mathrm{C}_{2}-\mathrm{H}\right) .{ }^{13} \mathrm{C} \mathrm{NMR}\left(\mathrm{CDCl}_{3}\right): \delta=17.82\left(\mathrm{CH}_{3}\right), 23.67\left(\mathrm{C}_{1},-\mathrm{H}\right), 25.38$ $\left(\mathrm{CH}_{3}\right), 119.64$ (C-5), 120.63 (C-3), 121.79 (C-7), 122.65 (C-2'), 127.16 (C-8), 128.62 (C-4a), 131.98 (C-3'), 132.42 (C-4), 143.14 (C-8a), 145.88 (C-2), 152.12 (C-6). Anal. Calcd for $\mathrm{C}_{14} \mathrm{H}_{15} \mathrm{NO}$ : C, 78.84; H, 7.09; N, 6.57. Found: C, 78.60; H, 6.87; N, 6.33.

Compound 8. Brown oil; $R_{f}=0.82\left(\mathrm{CHCl}_{3}-\right.$ acetone $\left.10: 1\right)$. IR (film): $1680(\mathrm{~s}, \mathrm{CO}) \mathrm{cm}^{-1} .{ }^{1} \mathrm{H}$ $\operatorname{NMR}\left(\mathrm{CDCl}_{3}\right): \delta=1.43\left(\mathrm{~s}, 6 \mathrm{H}, 2 \mathrm{CH}_{3}\right), 1.47\left(\mathrm{~s}, 6 \mathrm{H}, 2 \mathrm{CH}_{3}\right), 2.49\left(\mathrm{dd}, J=14\right.$ and $7 \mathrm{~Hz}, 2 \mathrm{H}, \mathrm{C}_{1}$ '$\mathrm{H}), 2.88\left(\mathrm{dd}, J=14\right.$ and $\left.7 \mathrm{~Hz}, 2 \mathrm{H}, \mathrm{C}_{1},-\mathrm{H}\right), 4.58$ (t, $\left.J=7 \mathrm{~Hz}, 2 \mathrm{H}, \mathrm{C}_{2},-\mathrm{H}\right), 6.36(\mathrm{~d}, J=10 \mathrm{~Hz}, 1 \mathrm{H}$, $\left.\mathrm{C}_{7}-\mathrm{H}\right), 7.29\left(\mathrm{dd}, J=7\right.$ and $\left.7.5 \mathrm{~Hz}, 1 \mathrm{H}, \mathrm{C}_{3}-\mathrm{H}\right), 7.57\left(\mathrm{~d}, J=10 \mathrm{~Hz}, 1 \mathrm{H}, \mathrm{C}_{8}-\mathrm{H}\right), 7.69(\mathrm{~d}, J=8 \mathrm{~Hz}, 1 \mathrm{H}$, $\left.\mathrm{C}_{4}-\mathrm{H}\right), 8.56\left(\mathrm{~d}, J=5 \mathrm{~Hz}, 1 \mathrm{H}, \mathrm{C}_{2}-\mathrm{H}\right) .{ }^{13} \mathrm{C} \mathrm{NMR}\left(\mathrm{CDCl}_{3}\right): \delta=17.83\left(\mathrm{CH}_{3}\right), 25.58\left(\mathrm{CH}_{3}\right), 56.02(\mathrm{C}-$ 5), 117.84 (C-2'), 123.19 (C-3), 129.81 (C-7), 134.24 (C-4), 134.90 (C-3'), 140.18 (C-4a), 145.97 (C-8), 147.98 (C-2), 149.85 (C-8a), 202.69 (C-6). Anal. Calcd for $\mathrm{C}_{19} \mathrm{H}_{23} \mathrm{NO}$ : C, 81.10; H, 8.24; N, 4.98. Found: C, 80.82; H, 7.95; N, 4.69 .

\section{5-(Cyclohex-2-en-1-yl)quinolin-6-ol (4d)}

A solution of $3 \mathbf{c}(1.0 \mathrm{~g}, 4.4 \mathrm{mmol})$ in chlorobenzene $(45 \mathrm{~mL})$ was stirred under reflux for $100 \mathrm{~h}$. The solvent was evaporated under reduced pressure and the residue was purified by column chromatography on silica gel $\left(\mathrm{CH}_{2} \mathrm{Cl}_{2}\right.$ - acetone $\left.10: 1\right)$ to yield $4 \mathbf{d}(0.7 \mathrm{~g}, 70 \%)$ as colorless crystals. Mp $148-152^{\circ} \mathrm{C} . R_{f}=0.27\left(\mathrm{CHCl}_{3}-\right.$ acetone $\left.10: 1\right)$.

IR (KBr): $3400(\mathrm{~s}, \mathrm{OH}) \mathrm{cm}^{-1} .{ }^{1} \mathrm{H}$ NMR $\left(\mathrm{CDCl}_{3}\right): \delta=1.84\left(\mathrm{~m}, 1 \mathrm{H}, \mathrm{C}_{5},-\mathrm{H}\right), 1.97\left(\mathrm{~m}, 2 \mathrm{H}, \mathrm{C}_{5},-\mathrm{H}\right.$ and $\left.\mathrm{C}_{6},-\mathrm{H}\right), 2.05\left(\mathrm{~m}, 1 \mathrm{H}, \mathrm{C}_{6},-\mathrm{H}\right), 2.20\left(\mathrm{~m}, 1 \mathrm{H}, \mathrm{C}_{4},-\mathrm{H}\right), 2.29\left(\mathrm{~m}, 1 \mathrm{H}, \mathrm{C}_{4},-\mathrm{H}\right), 4.51\left(\mathrm{~m}, 1 \mathrm{H}, \mathrm{C}_{1},-\mathrm{H}\right), 5.82$ $\left(\mathrm{m}, 1 \mathrm{H}, \mathrm{C}_{2},-\mathrm{H}\right), 5.88\left(\mathrm{~m}, 1 \mathrm{H}, \mathrm{C}_{3},-\mathrm{H}\right), 7.34\left(\mathrm{dd}, J=8.5\right.$ and $\left.4.0 \mathrm{~Hz}, 1 \mathrm{H}, \mathrm{C}_{3}-\mathrm{H}\right), 7.42(\mathrm{~d}, J=9.0 \mathrm{~Hz}$, $\left.1 \mathrm{H}, \mathrm{C}_{7}-\mathrm{H}\right), 7.80\left(\mathrm{~d}, J=9.0 \mathrm{~Hz}, 1 \mathrm{H}, \mathrm{C}_{8}-\mathrm{H}\right), 8.65\left(\mathrm{~d}, J=8.5 \mathrm{~Hz}, 1 \mathrm{H}, \mathrm{C}_{4}-\mathrm{H}\right), 8.66(\mathrm{~d}, J=4.0 \mathrm{~Hz}, 1 \mathrm{H}$, $\left.\mathrm{C}_{2}-\mathrm{H}\right), 8.8$ (br. s, $\left.1 \mathrm{H}, \mathrm{OH}\right) .{ }^{13} \mathrm{C} \mathrm{NMR}\left(\mathrm{CDCl}_{3}\right)$ : $\delta=24.12$ (C-5'), 25.57 (C-4'), 29.8 (C-6’9, 34.99 
(C-1'), 121.07 (C-3), 122.19 (C-7), 123.46 (C-5), 127.71 (C-3'), 129.25 (C-4a), 130.29 (C-8), 133.00 (C-4), 133.02 (C-2'), 145.85 (C-8a), 147.59 (C-2), 153.18 (C-6). Anal. Calcd for $\mathrm{C}_{15} \mathrm{H}_{15} \mathrm{NO}$ : C, 79.97; H, 6.71; N, 6.22. Found: C, 80.22; H, 6.95; N, 6.01.

\section{5-(Cyclohept-2-en-1-yl)quinolin-6-ol (4e)}

A solution of $\mathbf{3 d}(0.5 \mathrm{~g}, 2.1 \mathrm{mmol})$ in chlorobenzene $(20 \mathrm{~mL})$ was stirred under reflux for $100 \mathrm{~h}$. The solvent was evaporated under reduced pressure and the residue was purified by column chromatography on silica gel $\left(\mathrm{CH}_{2} \mathrm{Cl}_{2}-\right.$ acetone $\left.10: 1\right)$ to yield $4 \mathrm{e}(0.2 \mathrm{~g}, 38 \%)$ as colorless crystals. Mp 85-90 ${ }^{\circ}$ C. $R_{f}=0.42\left(\mathrm{CH}_{2} \mathrm{Cl}_{2}\right.$-acetone 4:1). IR $(\mathrm{KBr}): 3420(\mathrm{~s}, \mathrm{OH}) \mathrm{cm}^{-1}$.

${ }^{1} \mathrm{H} \mathrm{NMR}\left(\mathrm{CDCl}_{3}\right): \delta=1.50\left(\mathrm{~m}, 1 \mathrm{H}, \mathrm{C}_{5},-\mathrm{H}\right), 1.75\left(\mathrm{~m}, 1 \mathrm{H}, \mathrm{C}_{6},-\mathrm{H}\right), 1.88\left(\mathrm{~m}, 1 \mathrm{H}, \mathrm{C}_{7},-\mathrm{H}\right), 1.93(\mathrm{~m}$, $\left.1 \mathrm{H}, \mathrm{C}_{5},-\mathrm{H}\right), 2.08\left(\mathrm{~m}, 1 \mathrm{H}, \mathrm{C}_{6},-\mathrm{H}\right), 2.11\left(\mathrm{~m}, 1 \mathrm{H}, \mathrm{C}_{7},-\mathrm{H}\right), 2.27\left(\mathrm{~m}, 1 \mathrm{H}, \mathrm{C}_{4},-\mathrm{H}\right), 2.41\left(\mathrm{~m}, 1 \mathrm{H}, \mathrm{C}_{4},-\mathrm{H}\right)$, $4.62\left(\mathrm{~m}, 1 \mathrm{H}, \mathrm{C}_{1},-\mathrm{H}\right), 5.95\left(\mathrm{~m}, 2 \mathrm{H}, \mathrm{C}_{2},-\mathrm{H}\right.$ and $\left.\mathrm{C}_{3},-\mathrm{H}\right), 7.34\left(\mathrm{~d}, J=9.0 \mathrm{~Hz}, 1 \mathrm{H}, \mathrm{C}_{7}-\mathrm{H}\right), 7.36(\mathrm{dd}, J=$ 9.0 and $\left.4.2 \mathrm{~Hz}, 1 \mathrm{H}, \mathrm{C}_{3}-\mathrm{H}\right), 7.74\left(\mathrm{~d}, J=9.0 \mathrm{~Hz}, 1 \mathrm{H}, \mathrm{C}_{8}-\mathrm{H}\right), 8.56\left(\mathrm{~d}, J=8.4 \mathrm{~Hz}, 1 \mathrm{H}, \mathrm{C}_{4}-\mathrm{H}\right), 8.70$ $\left(\mathrm{dd}, J=4.2 \mathrm{~Hz}, 1 \mathrm{H}, \mathrm{C}_{2}-\mathrm{H}\right), 11.7$ (br. s, $\left.1 \mathrm{H}, \mathrm{OH}\right) .{ }^{13} \mathrm{C}$ NMR $\left(\mathrm{CDCl}_{3}\right): \delta=27.59(\mathrm{C}-5$ '), $29.58(\mathrm{C}-$ 4'), 31.24 (C-6'), 34.86 (C-7'), 38.36 (C-1'), 120.23 (C-3), 122.73 (C-7), 125.48 (C-5), 127.36 (C-8), 128.14 (C-4a), 131.65 (C-3’), 133.90 (C-4), 136.82 (C-2'), 143.14 (C-8a), 145.48 (C-2), 152.65 (C-6). Anal. Calcd for $\mathrm{C}_{16} \mathrm{H}_{17} \mathrm{NO}$ : C, 80.30; H, 7.16; N, 5.85. Found: C, 80.12; H, 7.32; $\mathrm{N}, 6.03$.

\section{Furo[3,2-f]quinolines (5a-e). General procedure}

A mixture of $4(2.5 \mathrm{mmol})$ and concd $\mathrm{H}_{2} \mathrm{SO}_{4}(0.25 \mathrm{~g}, 5.3 \mathrm{mmol})$ was heated in a water bath for $1.5 \mathrm{~h}$. After cooling, the reaction mixture was poured onto ice $(10 \mathrm{~g})$, basified with $1 \mathrm{~N} \mathrm{NaOH}(15$ $\mathrm{ml})$ and extracted with $\mathrm{CHCl}_{3}(3 \times 15 \mathrm{~mL})$. The combined organic layers were washed with $\mathrm{H}_{2} \mathrm{O}$, dried over $\mathrm{MgSO}_{4}$ and the solvent was evaporated in vacuo. The residue was purified by column chromatography on silica gel (using $\mathrm{CH}_{2} \mathrm{Cl}_{2}$ - acetone 5:1 as eluent).

1,2-Dihydro-2-methylfuro[3,2-flquinoline (5a). Yield: $35 \%$; brown oil, [lit., $\left.{ }^{2} 26.5^{\circ} \mathrm{C}\right], R_{f}=0.87$ $\left(\mathrm{CH}_{2} \mathrm{Cl}_{2}\right.$ - acetone 2:1). ${ }^{1} \mathrm{H} \mathrm{NMR}\left(\mathrm{CDCl}_{3}\right): \delta=1.56\left(\mathrm{~d}, J=6.3 \mathrm{~Hz}, 3 \mathrm{H}, \mathrm{CH}_{3}\right), 3.07(\mathrm{dd}, J=15.2$ and $\left.7.5 \mathrm{~Hz}, 1 \mathrm{H}, \mathrm{C}_{1}-\mathrm{H}\right), 3.60\left(\mathrm{dd}, J=15.2\right.$ and $\left.9.3 \mathrm{~Hz}, 1 \mathrm{H}, \mathrm{C}_{1}-\mathrm{H}\right), 5.17\left(\mathrm{~m}, 1 \mathrm{H}, \mathrm{C}_{2}-\mathrm{H}\right), 7.29(\mathrm{~d}, J=$ $\left.9 \mathrm{~Hz}, 1 \mathrm{H}, \mathrm{C}_{4}-\mathrm{H}\right), 7.35\left(\mathrm{dd}, J=8.4\right.$ and $\left.4.1 \mathrm{~Hz}, 1 \mathrm{H}, \mathrm{C}_{8}-\mathrm{H}\right), 7.90\left(\mathrm{~d}, J=8.3 \mathrm{~Hz}, 1 \mathrm{H}, \mathrm{C}_{9}-\mathrm{H}\right), 7.93(\mathrm{~d}$, $\left.J=9 \mathrm{~Hz}, 1 \mathrm{H}, \mathrm{C}_{5}-\mathrm{H}\right), 8.74\left(\mathrm{~d}, J=2.9,1 \mathrm{H}, \mathrm{C}_{7}-\mathrm{H}\right) .{ }^{13} \mathrm{C} \mathrm{NMR}\left(\mathrm{CDCl}_{3}\right): \delta=22.09\left(\mathrm{CH}_{3}\right), 35.56(\mathrm{C}-1)$, 80.88 (C-2), 115.39 (C-4), 118.20 (C-9b), 121.31 (C-8), 126.02 (C-9a), 130.33 (C-5), 130.88 (C9), 144.54 (C-5a), 147.16 (C-7), 157.20 (C-3a).

1,2-Dihydro-1,1,2-trimethylfuro[3,2-f]quinoline (5b). Yield: $42.2 \%$; light yellow oil; $R_{f}=0.44$ $\left(\mathrm{CHCl}_{3}\right.$ - acetone 10:1). ${ }^{1} \mathrm{H} \mathrm{NMR}\left(\mathrm{CDCl}_{3}\right): \delta=1.32\left(\mathrm{~s}, 3 \mathrm{H}, \mathrm{C}_{1}-\mathrm{CH}_{3}\right), 1.46\left(\mathrm{~d}, J=6.6 \mathrm{~Hz}, 3 \mathrm{H}, \mathrm{C}_{2}-\right.$ $\left.\mathrm{CH}_{3}\right), 1.62$ (s, 3H, $\left.\mathrm{C}_{1}-\mathrm{CH}_{3}\right), 4.53$ (q, $\left.J=6.6 \mathrm{~Hz}, 1 \mathrm{H}, \mathrm{C}_{2}-\mathrm{H}\right), 7.29$ (d, $\left.J=8.9 \mathrm{~Hz}, 1 \mathrm{H}, \mathrm{C}_{4}-\mathrm{H}\right), 7.33$ $\left(\mathrm{dd}, J=8.5\right.$ and $\left.4.0 \mathrm{~Hz}, 1 \mathrm{H}, \mathrm{C}_{8}-\mathrm{H}\right), 7.93\left(\mathrm{~d}, J=8.9 \mathrm{~Hz}, 1 \mathrm{H}, \mathrm{C}_{5}-\mathrm{H}\right), 8.29\left(\mathrm{~d}, J=8.5 \mathrm{~Hz}, 1 \mathrm{H}, \mathrm{C}_{9}-\mathrm{H}\right)$, $8.73\left(\mathrm{~d}, J=3.0 \mathrm{~Hz}, 1 \mathrm{H}, \mathrm{C}_{7}-\mathrm{H}\right) .{ }^{13} \mathrm{C} \mathrm{NMR}\left(\mathrm{CDCl}_{3}\right): \delta=13.89\left(\mathrm{CH}_{3}\right), 22.07\left(\mathrm{CH}_{3}\right), 26.56\left(\mathrm{CH}_{3}\right)$, 45.31 (C-1), 89.45 (C-2), 115.77 (C-4), 120.97 (C-8), 125.68 (C-9a), 126.89 (C-9b), 129.77 (C9), 130.60 (C-5), 145.15 (C-5a), 146.88 (C-7), 156.10 (C-3a). Anal. Calcd for $\mathrm{C}_{14} \mathrm{H}_{15} \mathrm{NO}$ : C, 78.84; H, 7.09; N, 6.57. Found: C, 79.07; H, 6.92; N, 6.33 
1,2-Dihydro-1,2,2-trimethylfuro[3,2-f $]$ quinoline (5c). Yield: 40\%; light yellow oil; $R_{f}=0.59$ $\left(\mathrm{CHCl}_{3}\right.$ - acetone 10:1). ${ }^{1} \mathrm{H} \mathrm{NMR}\left(\mathrm{CDCl}_{3}\right): \delta=1.31\left(\mathrm{~d}, J=7.1 \mathrm{~Hz}, 3 \mathrm{H}, \mathrm{C}_{1}-\mathrm{CH}_{3}\right), 1.44\left(\mathrm{~s}, 3 \mathrm{H}, \mathrm{C}_{2}-\right.$ $\mathrm{CH}_{3}$ ), 1.53 (s, 3H, $\mathrm{C}_{2}-\mathrm{CH}_{3}$ ), 3.44 (q, $\left.J=7.1 \mathrm{~Hz}, 1 \mathrm{H}, \mathrm{C}_{1}-\mathrm{H}\right), 7.27$ (d, J=8.9 Hz, 1H, C $\left.4-\mathrm{H}\right), 7.34$ (dd, $J=8.4$ and4.1 Hz, 1H, C $8-\mathrm{H}), 7.93$ (d, $\left.J=8.9 \mathrm{~Hz}, 1 \mathrm{H}, \mathrm{C}_{5}-\mathrm{H}\right), 8.04$ (d, $\left.J=8.4 \mathrm{~Hz}, 1 \mathrm{H}, \mathrm{C}_{9}-\mathrm{H}\right)$, $8.73\left(\mathrm{~d}, J=3.3 \mathrm{~Hz}, 1 \mathrm{H}, \mathrm{C}_{7}-\mathrm{H}\right) .{ }^{13} \mathrm{C} \mathrm{NMR}\left(\mathrm{CDCl}_{3}\right): \delta=16.37\left(\mathrm{C}_{1}-\mathrm{CH}_{3}\right), 22.41\left(\mathrm{C}_{2}-\mathrm{CH}_{3}\right), 28.52$ $\left(\mathrm{C}_{2}-\mathrm{CH}_{3}\right), 44.66$ (C-1), 90.23 (C-2), 115.96 (C-4), 121.13 (C-8), 123.90 (C-9b), 126.05 (C-9a), 130.36 (C-9), 130.44 (C-5), 144.80 (C-5a), 146.91 (C-7), 155.32 (C-3a). Anal. Calcd for $\mathrm{C}_{14} \mathrm{H}_{15} \mathrm{NO}$ : C, 78.84; H, 7.09; N, 6.57. Found: C, 79.01; H, 6.92; N, 6.30.

2,3-Dihydro-3,3-dimethyl-1H-pyrano[3,2-f]quinoline (9). Yield: 54\%; brown oil; $R_{f}=0.66$ $\left(\mathrm{CHCl}_{3}\right.$ - acetone 10:1). ${ }^{1} \mathrm{H}$ NMR $\left(\mathrm{CDCl}_{3}\right): \delta=1.39\left(\mathrm{~s}, 6 \mathrm{H}, 2 \mathrm{CH}_{3}\right), 1.95\left(\mathrm{~m}, 2 \mathrm{H}, \mathrm{C}_{2}-\mathrm{H}\right), 3.00(\mathrm{~m}$, $\left.2 \mathrm{H}, \mathrm{C}_{1}-\mathrm{H}\right), 7.25\left(\mathrm{~d}, J=9.6 \mathrm{~Hz}, 1 \mathrm{H}, \mathrm{C}_{5}-\mathrm{H}\right), 7.40\left(\mathrm{~m}, 1 \mathrm{H}, \mathrm{C}_{9}-\mathrm{H}\right), 7.91\left(\mathrm{~d}, J=9.0 \mathrm{~Hz}, 1 \mathrm{H}, \mathrm{C}_{6}-\mathrm{H}\right)$, $8.19\left(\mathrm{~d}, J=8.2 \mathrm{~Hz}, 1 \mathrm{H}, \mathrm{C}_{10}-\mathrm{H}\right), 8.74\left(\mathrm{~m}, 1 \mathrm{H}, \mathrm{C}_{8}-\mathrm{H}\right) .{ }^{13} \mathrm{C} \mathrm{NMR}\left(\mathrm{CDCl}_{3}\right): \delta=18.84(\mathrm{C}-1), 26.48$ $\left(\mathrm{CH}_{3}\right), 32.23$ (C-2), 74.53 (C-3), 112.08 (C-10b), 120.88 (C-9), 123.80 (C-5), 128.12 (C-10a), 128.45 (C-6), 130.77 (C-10), 143.51 (C-6a), 146.38 (C-8), 151.75 (C-4a).

7a,8,9,10,11,11a-Hexahydrobenzofuro[3,2-f]quinoline (5d). Yield: 75\%; yellow oil; ; $R_{f}=0.72$ $\left(\mathrm{CH}_{2} \mathrm{Cl}_{2}-\right.$ acetone $\left.2: 1\right)$. cis-Isomer. $t_{\mathrm{R}}=15.72 \mathrm{~min} .{ }^{1} \mathrm{H} \mathrm{NMR}\left(\mathrm{CDCl}_{3}\right): \delta=1.25\left(\mathrm{~m}, 1 \mathrm{H}, \mathrm{C}_{11}-\mathrm{H}\right)$, $1.32\left(\mathrm{~m}, 1 \mathrm{H}, \mathrm{C}_{10}-\mathrm{H}\right), 1.57\left(\mathrm{~m}, 1 \mathrm{H}, \mathrm{C}_{9}-\mathrm{H}\right), 1.67\left(\mathrm{~m}, 1 \mathrm{H}, \mathrm{C}_{10}-\mathrm{H}\right), 1.86\left(\mathrm{~m}, 1 \mathrm{H}, \mathrm{C}_{8}-\mathrm{H}\right), 2.12(\mathrm{~m}, 1 \mathrm{H}$, $\left.\mathrm{C}_{11}-\mathrm{H}\right), 2.35\left(\mathrm{~m}, 1 \mathrm{H}, \mathrm{C}_{8}-\mathrm{H}\right), 3.45\left(\mathrm{~m}, 1 \mathrm{H}, \mathrm{C}_{11 \mathrm{a}}-\mathrm{H}\right), 4.82\left(\mathrm{~m}, 1 \mathrm{H}, \mathrm{C}_{7 \mathrm{a}}-\mathrm{H}\right), 7.32(\mathrm{dd}, J=8.5$ and 4.2 $\left.\mathrm{Hz}, 1 \mathrm{H}, \mathrm{C}_{2}-\mathrm{H}\right), 7.34$ (d, $\left.J=9 \mathrm{~Hz}, 1 \mathrm{H}, \mathrm{C}_{6}-\mathrm{H}\right), 7.93$ (d, $\left.J=9 \mathrm{~Hz}, 1 \mathrm{H}, \mathrm{C}_{5}-\mathrm{H}\right), 7.99$ (d, J=8.2 Hz, 1H, $\left.\mathrm{C}_{1}-\mathrm{H}\right), 8.73\left(\mathrm{~d}, J=3 \mathrm{~Hz}, 1 \mathrm{H}, \mathrm{C}_{3}-\mathrm{H}\right) .{ }^{13} \mathrm{C} \mathrm{NMR}\left(\mathrm{CDCl}_{3}\right): \delta=20.18(\mathrm{C}-9), 22.37(\mathrm{C}-10), 27.24(\mathrm{C}-$ 8), 29.19 (C-11), 39.59 (C-11a), 84.00 (C-7a), 115.85 (C-6), 121.20 (C-2), 125.35 (C-11c), 126.48 (C-11b), 129.89 (C-5), 130.88 (C-1), 144.66 (C-4a), 147.19 (C-3), 156.93 (C-6a).

Anal. Calcd for $\mathrm{C}_{15} \mathrm{H}_{15} \mathrm{NO}$ : C, 79.97; H, 6.71; N, 6.22.Found: C, 80.25; H, 6.98; N, 6.00.

trans-Isomer. $t_{\mathrm{R}}=13.37 \mathrm{~min} .{ }^{1} \mathrm{H}$ NMR $\left(\mathrm{CDCl}_{3}\right): \delta=1.32\left(\mathrm{~m}, 1 \mathrm{H}, \mathrm{C}_{9}-\mathrm{H}\right), 1.50\left(\mathrm{~m}, 1 \mathrm{H}, \mathrm{C}_{9}-\mathrm{H}\right)$, $1.67\left(\mathrm{~m}, 1 \mathrm{H}, \mathrm{C}_{8}-\mathrm{H}\right), 1.88\left(\mathrm{~m}, 2 \mathrm{H}, \mathrm{C}_{10}-\mathrm{H}\right), 2.03\left(\mathrm{~m}, 2 \mathrm{H}, \mathrm{C}_{11}-\mathrm{H}\right), 2.15$ (m, 1H, $\left.\mathrm{C}_{8}-\mathrm{H}\right), 3.62$ (br. s, $\left.1 \mathrm{H}, \mathrm{C}_{11 \mathrm{a}}-\mathrm{H}\right), 4.68$ (br. s, $\left.1 \mathrm{H}, \mathrm{C}_{7 \mathrm{a}}-\mathrm{H}\right), 7.30\left(\mathrm{~d}, J=9.1 \mathrm{~Hz}, 1 \mathrm{H}, \mathrm{C}_{6}-\mathrm{H}\right), 7.34((\mathrm{dd}, J=8.5$ and $4.0 \mathrm{~Hz}$, $\left.1 \mathrm{H}, \mathrm{C}_{2}-\mathrm{H}\right), 7.88\left(\mathrm{~d}, J=9.1 \mathrm{~Hz}, 1 \mathrm{H}, \mathrm{C}_{5}-\mathrm{H}\right), 8.15\left(\mathrm{~d}, J=8.5 \mathrm{~Hz}, 1 \mathrm{H}, \mathrm{C}_{1}-\mathrm{H}\right), 8.72(\mathrm{~d}, J=3 \mathrm{~Hz}, 1 \mathrm{H}$, $\left.\mathrm{C}_{3}-\mathrm{H}\right) .{ }^{13} \mathrm{C}$ NMR $\left(\mathrm{CDCl}_{3}\right): \delta=17.86(\mathrm{C}-9), 26.33$ (C-11a), $29.20(\mathrm{C}-11), 31.06(\mathrm{C}-10), 33.79(\mathrm{C}-$ 8), 70.84 (C-7a), 116.83 (C-11b), 120.94 (C-2), 121.67 (C-6), 126.92 (C-11c), 128.97 (C-5), 129.56 (C-1), 144.22 (C-4a), 146.83 (C-3), 153.90 (C-6a). Anal. Calcd for $\mathrm{C}_{15} \mathrm{H}_{15} \mathrm{NO}$ : C, 79.97; H, 6.71; N, 6.22. Found: C, 80.17; H, 6.99; N, 5.98.

\section{$\mathbf{8 , 9 , 1 0 , 1 1 , 1 2 , 1 2 a - h e x a h y d r o - 7 a} \boldsymbol{H}$-cycloheptafuro[3,2-f]quinoline (5e)}

Yield: $74 \% ; R_{f}=0.71\left(\mathrm{CH}_{2} \mathrm{Cl}_{2}-\right.$ acetone $\left.4: 1\right)$.

cis-Isomer. $t_{R}=7.78 \mathrm{~min} .{ }^{1} \mathrm{H}$ NMR $\left(\mathrm{CDCl}_{3}\right): \delta=1.45\left(\mathrm{~m}, 1 \mathrm{H}, \mathrm{C}_{9}-\mathrm{H}\right), 1.50\left(\mathrm{~m}, 2 \mathrm{H}, \mathrm{C}_{10}-\mathrm{H}\right.$ and $\left.\mathrm{C}_{11}-\mathrm{H}\right), 1.71\left(\mathrm{~m}, 1 \mathrm{H}, \mathrm{C}_{11}-\mathrm{H}\right), 1.75\left(\mathrm{~m}, 1 \mathrm{H}, \mathrm{C}_{10}-\mathrm{H}\right), 1.89\left(\mathrm{~m}, 2 \mathrm{H}, \mathrm{C}_{9}-\mathrm{H}\right.$ and $\left.\mathrm{C}_{12}-\mathrm{H}\right), 1.94(\mathrm{~m}, 1 \mathrm{H}$, $\left.\mathrm{C}_{12}-\mathrm{H}\right), 2.08\left(\mathrm{~m}, 1 \mathrm{H}, \mathrm{C}_{8}-\mathrm{H}\right), 2.27\left(\mathrm{~m}, 1 \mathrm{H}, \mathrm{C}_{8}-\mathrm{H}\right), 3.87\left(\mathrm{~m}, 1 \mathrm{H}, \mathrm{C}_{12 \mathrm{a}}-\mathrm{H}\right), 5.14\left(\mathrm{~m}, 1 \mathrm{H}, \mathrm{C}_{7 \mathrm{a}}-\mathrm{H}\right), 7.27$ $\left(\mathrm{d}, J=8.0 \mathrm{~Hz}, 1 \mathrm{H}, \mathrm{C}_{6}-\mathrm{H}\right), 7.32\left(\mathrm{dd}, J=8.5\right.$ and $\left.4.2 \mathrm{~Hz}, 1 \mathrm{H}, \mathrm{C}_{2}-\mathrm{H}\right), 7.92\left(\mathrm{~d}, J=8.0 \mathrm{~Hz}, 1 \mathrm{H}, \mathrm{C}_{5}-\mathrm{H}\right)$, $8.03\left(\mathrm{~d}, J=8.3 \mathrm{~Hz}, 1 \mathrm{H}, \mathrm{C}_{1}-\mathrm{H}\right), 8.72\left(\mathrm{dd}, J=3.7\right.$ and $\left.1.0 \mathrm{~Hz}, 1 \mathrm{H}, \mathrm{C}_{3}-\mathrm{H}\right) .{ }^{13} \mathrm{C} \mathrm{NMR}\left(\mathrm{CDCl}_{3}\right): \delta=$ 23.67 (C-9), 28.84 (C-11), 30.01 (C-12), 31.21 (C-10), 31.45 (C-8), 46.47 (C-12a), 88.19 (C-7a), 115.23 (C-6), 121.12 (C-2), 122.59 (C-5), 125.69 (C-12b), 130.32 (C-1), 130.67 (C-5), 144.89 
(C-4a), 146.95 (C-3), 156.63 (C-6a). Anal. Calcd for $\mathrm{C}_{16} \mathrm{H}_{17} \mathrm{NO}$ : C, 80.30; H, 7.16; N, 5.85. Found: C, 80.10; H, 7.38; N, 6.04.

trans-Isomer. $t_{R}=7.08 \mathrm{~min} .{ }^{1} \mathrm{H}$ NMR $\left(\mathrm{CDCl}_{3}\right): \delta=1.34\left(\mathrm{~m}, 1 \mathrm{H}, \mathrm{C}_{11}-\mathrm{H}\right), 1.48\left(\mathrm{~m}, 1 \mathrm{H}, \mathrm{C}_{9}-\mathrm{H}\right)$, $1.57\left(\mathrm{~m}, 1 \mathrm{H}, \mathrm{C}_{11}-\mathrm{H}\right), 1.93\left(\mathrm{~m}, 1 \mathrm{H}, \mathrm{C}_{8}-\mathrm{H}\right), 2.10\left(\mathrm{~m}, 3 \mathrm{H}, \mathrm{C}_{8}-\mathrm{H}, \mathrm{C}_{10}-\mathrm{H}\right.$ and $\left.\mathrm{C}_{12}-\mathrm{H}\right), 2.36(\mathrm{~m}, 1 \mathrm{H}$, $\left.\mathrm{C}_{10}-\mathrm{H}\right), 3.68$ (br. s, 1H, $\left.\mathrm{C}_{12 \mathrm{a}}-\mathrm{H}\right), 4.82$ (br. s, $\left.1 \mathrm{H}, \mathrm{C}_{7 \mathrm{a}}-\mathrm{H}\right), 7.24$ (d, J=9.2 Hz, 1H, C 6 -H), 7.36 ((dd, $J=8.5$ and $\left.4.2 \mathrm{~Hz}, 1 \mathrm{H}, \mathrm{C}_{2}-\mathrm{H}\right), 7.86\left(\mathrm{~d}, J=9.2 \mathrm{~Hz}, 1 \mathrm{H}, \mathrm{C}_{5}-\mathrm{H}\right), 8.17\left(\mathrm{~d}, J=8.5 \mathrm{~Hz}, 1 \mathrm{H}, \mathrm{C}_{1}-\mathrm{H}\right), 8.73$ $\left(\mathrm{d}, J=3.3 \mathrm{~Hz}, 1 \mathrm{H}, \mathrm{C}_{3}-\mathrm{H}\right) .{ }^{13} \mathrm{C} \mathrm{NMR}\left(\mathrm{CDCl}_{3}\right): \delta=23.77(\mathrm{C}-9), 25.67$ (C-11), $27.50(\mathrm{C}-12 \mathrm{a}), 28.43$ (C-10), 34.63 (C-12), 36.89 (C-8), 73.03 (C-7a), 117.05 (C-12b), 120.78 (C-2), 122.64 (C-6), 127.14 (C-12c), 129.20 (C-5), 130.30 (C-1), 144.61 (C-4a), 146.71 (C-3), 151.84 (C-6a). Anal. Calcd for $\mathrm{C}_{16} \mathrm{H}_{17} \mathrm{NO}$ : C, 80.30; H, 7.16; N, 5.85. Found: C, 80.06; H, 7.28; N, 6.07.

\section{Acknowledgements}

This work was supported by the Hungarian OTKA Foundation (T 42515) and NKFP program contract MediChem II (Hungarian Ministry of Education). We would like to thank Csilla Hegyi and Sarolta Pilbak for the HPLC separations.

\section{References and Notes}

1. Dey, B. B.; Seshadri, T. R. Quart. J. Indian Chem. Soc. 1926, 3, 166.

2. Péne, C.; Demerseman, P.; Cheutin, A.; Royer, R. Bull. Soc. Chim. Fr.1966, 586.

3. Muratake, H.; Hayakawa, A.; Natsume, M. Tetrahedron Lett. 1997, 38, 7577.

4. Tőrincsi, A.; Kolonits, P.; Pálosi, E.; Novák, L. Synthesis 2007, 284.

5. Microwave -assisted organic reactions have gained considerable attention over the past decade, and the method also found application in preparing pharmaceutically active agents. See: Farand, J. A.; Denissova, I.; Barriault, L. Heterocycles 2004, 62, 735. Kotha, S.; Mandal, K.; Deb, A. Ch.; Banerjee, S. Tetrahedron Lett. 2004, 45, 9603. Rao, V. V.;Reddy, G. V.; Yadla, R.; Narsaiah, B.; Rao, P. S. Arkivoc 2005, 3, 211. Jacob, A. M.; Moody, Ch. J. Tetrahedron Lett. 2005, 46, 8823. Kremsner, J. M.; Kappe, C. O. J. Org. Chem. 2006, 71, 4651 , and references cited therein.

6. For excellent monograph on Claisen rearrangement, see: Tarbell, D. S. Org. React. 1944, 2, 2. Zigler, F. E. Acc. Chem. Res. 1977, 10, 227. Murray, A. W. Org. React. Mech. $1980,517$. Lutz, R. P. Chem. Rev. 1984, 84, 205. Zigler, F. E. Chem. Rev. 1988, 88, 1423. Nubbemeyer, U. Synthesis 2003, 961. Castro, A. M. M. Chem. Rev. 2004, 104, 2939.

7. This type of rearrangement $(\mathbf{3 b} \rightarrow \mathbf{4 c})$ was first reported by W.M. Lauer and W.F. Filbert: $J$. Am. Chem. Soc. 1936, 58, 1388. The mechanism of the reactions has been identified as the result of three consecutive processes: [3,3] rearrangement followed by 1,5-hydrogen shifts. Hurd, C. D. ; Pollack, M. A. J. Org. Chem. 1939, 3, 550. Marwell, E. N. J. Org. Chem. 
1960, 25, 676. Lauer, W. M.; Douldoras, G. A.; Hileman, R. E.; Lupino, R. J. Org. Chem. 1961, 26, 4785. Marwell, E. N.; Anderson, D. R.; Ong, J. J. Org. Chem. 1962, 27, 1109. Roberts, R. M.; Landolt, R. G.; Greene, R. N.; Heyer, E. W. J. Am. Chem. Soc.1967, 89, 1404. Scheinmann, F.; Barner, R.; Schmid, H. Helv. Chim. Acta 1968, 51, 1603. Rhoads, S. J.; Raulins, N. R. Org. React. 1974, 22, 1. Schobert, R.; Siegfried, S.; Gordon, G.; Mulholland, D.; Nieuwenhuyzen, M. Terahedron Lett. 2001, 42, 4561.

8. Cox, R. A.; Swallow, A. J. J. Chem. Soc. 1958, 3727.

9. Dondas, H. A.; Grigg, R.; Thibault, S. Tetrahedron 2001, 57, 7035. 